\title{
Time-Specific Contribution of the Supplementary Motor Area to Intermanual Transfer of Procedural Knowledge
}

\author{
Monica A. Perez, ${ }^{1}$ Satoshi Tanaka, ${ }^{1}$ Steven P. Wise, ${ }^{2}$ Daniel T. Willingham, ${ }^{3}$ and Leonardo G. Cohen ${ }^{1}$ \\ ${ }^{1}$ Human Cortical Physiology Section and Stroke Neurorehabilitation Clinic, National Institute of Neurological Disorders and Stroke-National Institutes of \\ Health, ${ }^{2}$ Laboratory of Systems Neuroscience, National Institute of Mental Health-National Institutes of Health, Bethesda, Maryland 20892, and \\ ${ }^{3}$ Department of Psychology, University of Virginia, Charlottesville, Virginia 22904
}

The supplementary motor area (SMA) makes a crucial contribution to intermanual transfer: the ability to use one hand to perform a skill practiced and learned with the other hand. However, the timing of this contribution relative to movement remains unknown. Here, 33 healthy volunteers performed a 12 item sequence in the serial reaction time task. During training, each participant responded to a sequence of visual cues presented at $1 \mathrm{~Hz}$ by pressing one of four keys with their right hand. The measure of intermanual transfer was response time (RT) during repetition of the trained sequence with the left hand, which was at rest during learning. Participants were divided into three groups, which did not differ in their learning rates or amounts. In two groups, $1 \mathrm{~Hz}$ repetitive transcranial magnetic stimulation induced transient virtual lesions of the SMA during training, either $100 \mathrm{~ms}$ before each cue (the premovement group) or during each key press (the movement group). The third group received sham stimulation (the sham group). After training with the right hand, RTs for performance with the left (transfer) hand were longer for the premovement group than for the movement or sham groups. Thus, the most crucial contribution of SMA to intermanual transfer occurs in the interval between movements, when the memory of a previous movement plays a role in encoding specific sequences. These results provide insight into frontal lobe contributions to procedural knowledge.

Key words: motor learning; training; plasticity; motor cortex; motor control; learning and memory

\section{Introduction}

The supplementary motor area (SMA) contributes to the preparation and execution of learned motor sequences (Shibasaki et al., 1993; Jenkins et al., 1994; Nakamura et al., 1998; Shima and Tanji, 1998; Lee and Quessy, 2003; Macar et al., 2004; Elsinger et al., 2006). It has been proposed that SMA activity during the interval between individual movements contributes to encoding and planning the next element in a motor sequence (Tanji and Shima, 1994; Gerloff et al., 1997; Shima and Tanji, 1998), whereas SMA activity during movement contributes to on-line control and monitoring of the movement (Seitz and Roland, 1992; Tanji and Shima, 1994; Shima and Tanji, 2000; Padoa-Schioppa et al., 2002; Lee and Quessy, 2003).

For sequence learning, as with other skills, the procedural knowledge acquired with one hand transfers to the other hand, a process called intermanual transfer (Parlow and Kinsbourne, 1989; Howard and Howard, 1997; Rand et al., 1998; Grafton et al., 2002; Japikse et al., 2003; Perez et al., 2007a,b). This process may occur implicitly (i.e., in the absence of awareness), and it is critical

\footnotetext{
Received July 21, 2008; revised Aug. 12, 2008; accepted Aug. 15, 2008.

This work was supported in part by the Intramural Research Program of the National Institutes of Health, National Institute of Neurological Disorders and Stroke, and National Institute of Mental Health.

Correspondence should be addressed to Leonardo G. Cohen, Human Cortical Physiology Section, National Institute of Neurological Disorders and Stroke-National Institutes of Health, 10 Center Drive, Bethesda, MD 20892-1430. E-mail: cohenl@ninds.nih.gov.

DOI:10.1523/JNEUROSCI.3416-08.2008

Copyright $\odot 2008$ Society for Neuroscience $\quad 0270-6474 / 08 / 289664-06 \$ 15.00 / 0$
}

to activities of daily life (Obayashi, 2004; Halsband and Lange, 2006). We showed previously that efficient intermanual transfer is associated with increased SMA activity and that transfer is blocked by repetitive transcranial magnetic stimulation (rTMS) over SMA (Perez et al., 2007b). However, the timing of the contribution of SMA to intermanual transfer remains unknown. Accordingly, here we evaluated the effect on intermanual transfer of disrupting SMA activity at two different times, during the interval between training movements and during the movements, as participants learned a motor sequence skill in the serial reaction time task (SRTT).

\section{Materials and Methods}

Subjects. Thirty-three healthy volunteers (14 females; 19 males) with a mean $( \pm S D)$ age of $28 \pm 5.4$ years participated in the study. All subjects gave their informed consent to the experimental procedure, which was approved by the National Institute of Neurological Disorders and Stroke (NINDS) ethics committee. The study was performed in accordance with the Declaration of Helsinki at the Human Cortical Physiology Section Laboratory, NINDS-National Institutes of Health. All subjects were right-handed and trained with the right hand for $\sim 30 \mathrm{~min}$ on the SRTT. During SRTT training, subjects were randomly assigned to three different stimulation groups for $1 \mathrm{~Hz}$ rTMS over the SMA: (1) rTMS during individual finger key presses (movement group; $n=11$ ), (2) rTMS preceding the cue and each individual finger key press (premovement group; $n=11$ ), and (3) sham stimulation with a time course matching the premovement group (sham group; $n=11$ ).

SRTT and transfer test. Subjects were seated in an armchair with both arms flexed at the elbow by $90^{\circ}$ and with the wrist in a neutral position. 
A

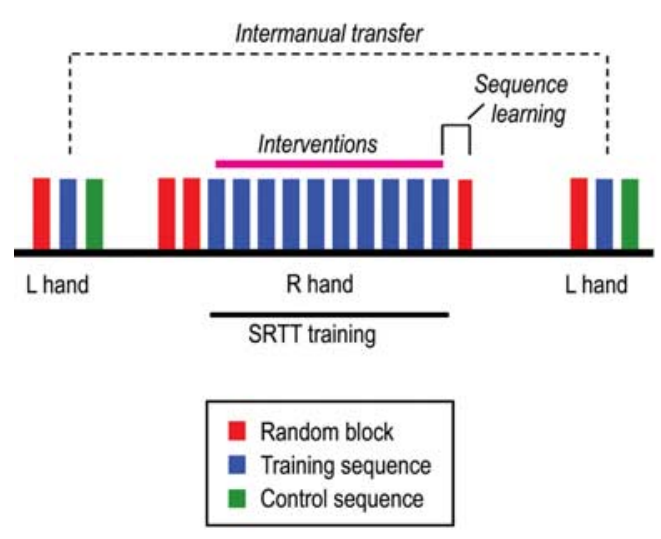

B

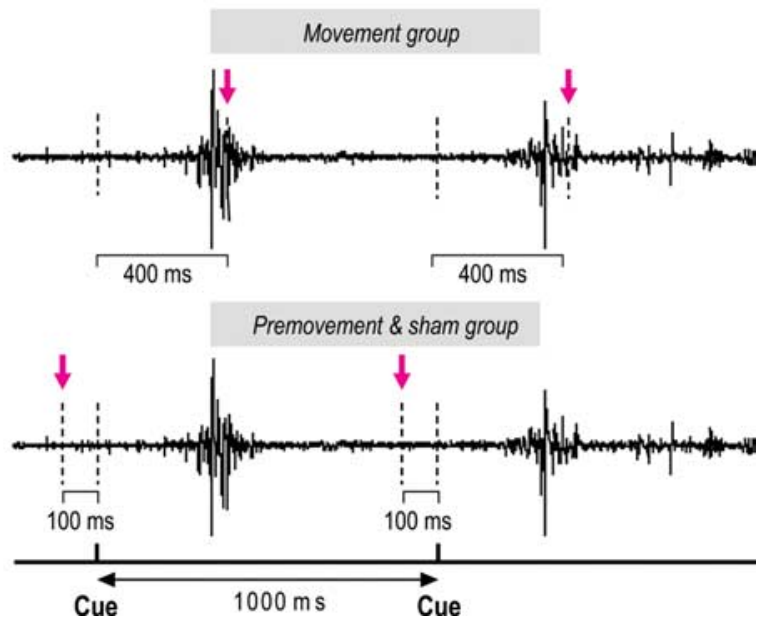

Figure 1. Experimental design. $A$, Order of the trial blocks performed with the left $(\mathrm{L})$ and right $(\mathrm{R})$ hands. Training sequence (blue), control sequence (green), and random (red) blocks were tested for the left hand, before and after practicing the SRTT with the right hand. Subjects performed 10 training sequence blocks, preceded by two random blocks and followed by one random block. $\boldsymbol{B}$, During SRTT training, three groups of subjects received rTMS at $1 \mathrm{~Hz}$ over the SMA (arrows): (1) during each finger key press (movement group), (2) before each each key press (premovement group), and (3) sham stimulation (sham group), which occurred at a time matching rTMS in the premovement group.

SRTT (Nissen and Bullemer, 1987) was presented on a computer screen using a custom script based on Presentation software (Neurobehavioral Systems). As illustrated in Figure $1 A$, subjects first performed three kinds of trial blocks with the left hand: (1) the mirror image of the training sequence, (2) a different, unlearned sequence (control sequence), and (3) a random block. The GO signal was displayed on a computer screen (one asterisk and three dots evenly spaced horizontally, all appearing simultaneously). The position of the asterisk, which served as the cue for which key to press, varied among the four possible locations each time a display appeared. The order of keys in the training sequence was 4-2-3-1-1-3-2$1-3-4-2-4$, and in the control sequence it was 1-3-3-4-2-1-3-2-4-1-4-2. In the random blocks, the asterisk appeared in a randomly selected series of locations. Subsequently, subjects proceeded to SRTT training (Fig. 1A) with their right hand. Here, subjects performed two random blocks followed by 10 training-sequence blocks and ending with another random block. During all testing, subjects were instructed to respond as fast and accurately as possible to the asterisk presented every $1.0 \mathrm{~s} \mathrm{(a} \mathrm{total} \mathrm{of} 120$ key presses per block). Response times (RTs) were measured and defined as the time interval between the GO signal and the correct key press. We also measured the difference in RT before and after training in the left hand $(\Delta \mathrm{RT})$ in all testing blocks. Intermanual transfer of sequencespecific learning was reflected by the magnitude of $\Delta \mathrm{RT}$ in the training sequence block, most importantly in comparison with $\Delta \mathrm{RT}$ in the random block. After SRTT training, subjects were tested again with their left hand. At the end of the experiments, subjects were asked: "Did you notice anything special about the task?" and "Do you have anything to report regarding the task?” Subjects who reported that they noticed a sequence or a repeating pattern of targets were asked to describe it in as much detail as possible using the keyboard, and that information was recorded.

Transcranial magnetic stimulation localization. Transcranial magnetic stimulation (TMS) was delivered from a Magstim rapid stimulator (Rapid Magstim) through a figure-of-eight coil (loop diameter, $8 \mathrm{~cm}$; type no. SP15560). At the beginning of each experiment, we determined the right primary motor cortex (M1) resting motor threshold (RMT), defined as the lowest intensity of TMS output required to elicit motorevoked potentials (MEPs) of at least $50 \mu \mathrm{V}$ in peak-to-peak amplitude in at least three of five consecutive trials (Rossini et al., 1994). The coil was placed over this site tangential to the scalp with the junction region pointing backwards and laterally at a $45^{\circ}$ angle away from the midline, inducing a posterior-anterior current in the brain (Di Lazzaro et al., 2004). To determine the site for SMA stimulation, we used a criteria previously described (Matsunaga et al., 2005). We determined the optimal position for activation of the left tibialis anterior (TA) muscle by moving the coil in $1 \mathrm{~cm}$ steps along the sagittal midline around scalp vertex $(\mathrm{Cz})$ with the handle pointing to the right. The active motor threshold (AMT) for this muscle was determined, and stimuli of $\sim 130 \%$ AMT were given, moving the coil anteriorly along the sagittal midline in $1 \mathrm{~cm}$ steps. The SMA was defined as being $1 \mathrm{~cm}$ anterior to the last site from which MEPs could be evoked during the contraction. In most subjects, following these criteria, the site for the SMA stimulation was determined to be $-3 \mathrm{~cm}$ anterior from the optimal position for activation of the TA muscle, $2-4 \mathrm{~cm}$ anterior to $\mathrm{Cz}$. For sham stimulation, the coil was positioned in the same location described above for the SMA. A second coil was located behind the subject's head and it discharged in the air using the same parameters described below.

$r$ TMS. rTMS was delivered over SMA to three different groups of subjects (11 subjects per group) during SRTT training (Fig. $1 B$ ) at a frequency of $1 \mathrm{~Hz}$ at $80 \%$ of RMT. TMS pulses were applied 120 times per block, producing a total of 1200 pulses. In one session, each rTMS pulse was delivered $400 \mathrm{~ms}$ after the presentation of the GO signal, which was the cue instructing a given key press. This time was determined in a pilot study $(n=7)$ by using a custom-made Signal script to acquire on-line electromyographic (EMG) activity from the right first dorsal interosseous (FDI) muscle during right index finger key presses for SRTT training. In the present study, EMG signals were rectified and averaged on-line after each training block. Reaction time (i.e., onset time of right FDI EMG activity in the first random block and last training sequence block; beginning of training, $196 \pm 28$; end of training, $119 \pm 26$ ) and movement time (i.e., duration of right FDI EMG burst; beginning of training, $334 \pm 7.3$; end of training, $320 \pm 5$ ) measurements indicated that, at 400 $\mathrm{ms}$, rTMS pulses were applied within the movement phase of individual finger key presses. In another session, each rTMS pulse was delivered 100 $\mathrm{ms}$ before the cue. Sham stimulation was applied $100 \mathrm{~ms}$ before the cue. In additional experiments, we tested the effect of rTMS $(n=11)$ and sham rTMS $(n=11)$ over the SMA in the premovement phase $(100 \mathrm{~ms}$ before the cue) on transfer on the original (non-mirror image) sequence. In this form of the task, the transfer hand targets the same spatial goals, as opposed to the mirror image form, in which transfer is movement based. The order of keys in the original sequence was 4-2-3-1-1-3-2-1-3-4-2-4 (little finger for key 1 , ring finger for key 2 , middle finger for key 3 , and index finger for key 4).

EMG recordings. EMG surface electrodes were positioned on the skin overlying the left and right FDI and left TA muscles in a bipolar montage (interelectrode distance, $2 \mathrm{~cm}$ ). The EMG signals were amplified, filtered (bandpass, $25 \mathrm{~Hz}$ to $1 \mathrm{kHz}$ ), sampled at $2 \mathrm{kHz}$, and stored on a personal computer for off-line analysis. 
Statistics. Repeated-measure ANOVA $\left(\mathrm{ANOVA}_{\mathrm{RM}}\right)$ test was used to evaluate the median RT and the number of errors performed with the right hand in the training sequence and random blocks for each group. A paired $t$ test was used to evaluate sequence learning with the right hand by comparing the median RT between the last training sequence block and the last random block (Willingham et al., 2000). One-way ANOVA $_{\mathrm{RM}}$ was used to compare sequence learning between the three groups with stimulation time (movement, premovement, and sham) as factor. We assessed rTMS effects on intermanual transfer with left-hand blocks (training sequence, control sequence, and random block) and testing time (before vs after training) as factors on each group. Two-way ANOVA $_{\mathrm{RM}}$ was used to assess rTMS effects on intermanual transfer with stimulation time (movement, premovement, and sham) and left-hand block type (training sequence, control sequence, and random block) as factors. Bonferroni's corrections were applied for multiple comparisons. Significance was set at $p<0.05$. Variance is expressed as mean $\pm \mathrm{SD}$, unless otherwise noted.

\section{Results}

All subjects completed the SRTT training with the right hand $(N=33)$. Seven of the 33 subjects distributed over the two stimulation and sham groups reported that some targets repeated during the SRTT training, but none was able to reproduce the entire sequence or triplet pairs within the sequence. rTMS stimulation intensity (expressed as a percentage of the stimulator output) was similar across groups (movement, $47.1 \pm 2 \%$; premovement, $43.8 \pm 3.1 \%$; sham, $46.2 \pm 4 \% ; F=0.7$; $p=0.5)$.

\section{Right (training) hand}

As shown Figure $2 \mathrm{~A}$, left, SRTT training led to progressively shorter RTs in the training sequence in all groups (movement: $\mathrm{ANOVA}_{\mathrm{RM}}, F=15.5, p \leq 0.001$; premovement: ANO$\mathrm{VA}_{\mathrm{RM}}, F=14.8, p \leq 0.001$; sham: $\mathrm{ANOVA}_{\mathrm{RM}}, F=14.3, p \leq$ $0.001)$. ANOVA $\mathrm{RM}_{\mathrm{RM}}$ showed that the magnitude of sequence learning (movement: $77.3 \pm 7.6 \mathrm{~ms}$; premovement: $83.6 \pm 7.3 \mathrm{~ms}$; sham: $73.5 \pm 5.7 \mathrm{~ms}$; $\left.\mathrm{ANOVA}_{\mathrm{RM}}, F=0.41, p=0.6\right)$ and the number of errors performed with the right hand (ANOVA $\mathrm{RM}_{\mathrm{RM}}$, $F=0.6, p=0.5$ ) were comparable for the training sequence and random blocks across groups.

\section{Left (transfer) hand}

Figure $2 B$, right, shows $\Delta \mathrm{RT}$ s for the left hand, comparing performance before and after SRTT training with the right hand. Training always involved the training sequence, but testing of left-hand performance involved three types of blocks: random (red), training sequence (blue), and control sequence (green). Larger values of $\Delta \mathrm{RT}$ indicate greater intermanual transfer, and data from the three rTMS groups (movement, premovement, and sham) appear from top to bottom.

For the movement group, ANOVA $_{\mathrm{RM}}$ showed a significant
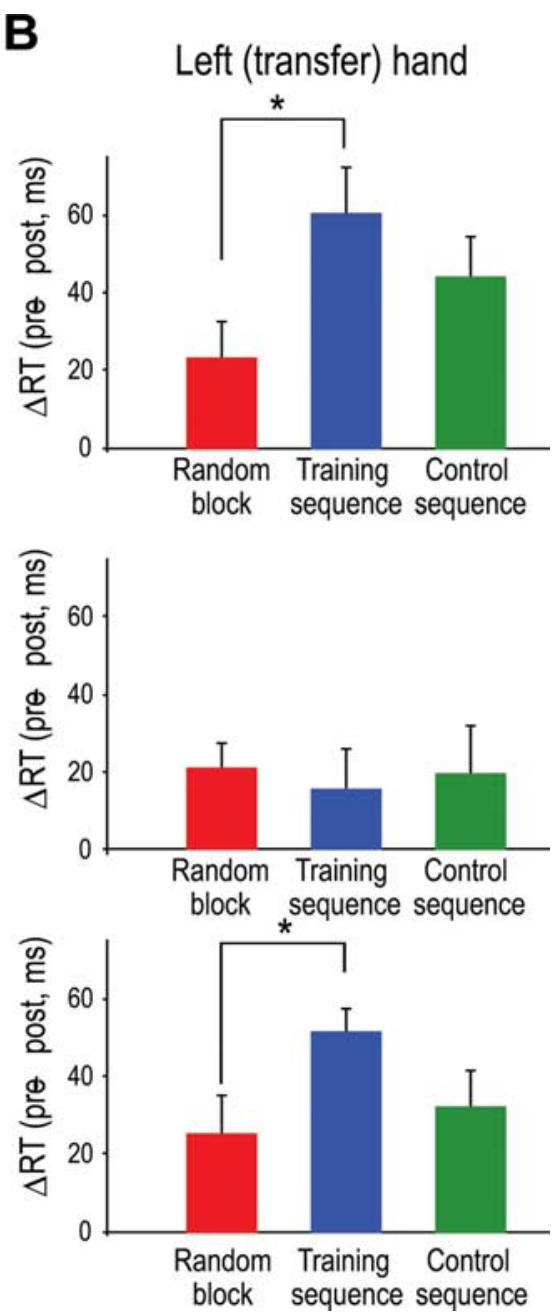

Figure 2. Learning and intermanual transfer of learning. $\boldsymbol{A}$, The abscissa shows the sequence of training blocks, and the ordinate shows RT for the right hand. Note the progressive shortening of RT with repeated performance of the training sequence ) in all three groups of subjects. The red bars show blocks with a random sequence. Significant sequence learning is shown by ormance after (vs before) right-hand training. The abscissa shows the type of block performed by the left hand: movement (top) and sham groups (bottom), indicating intermanual transfer, but not in the premovement group (middle). Variance, SEM.
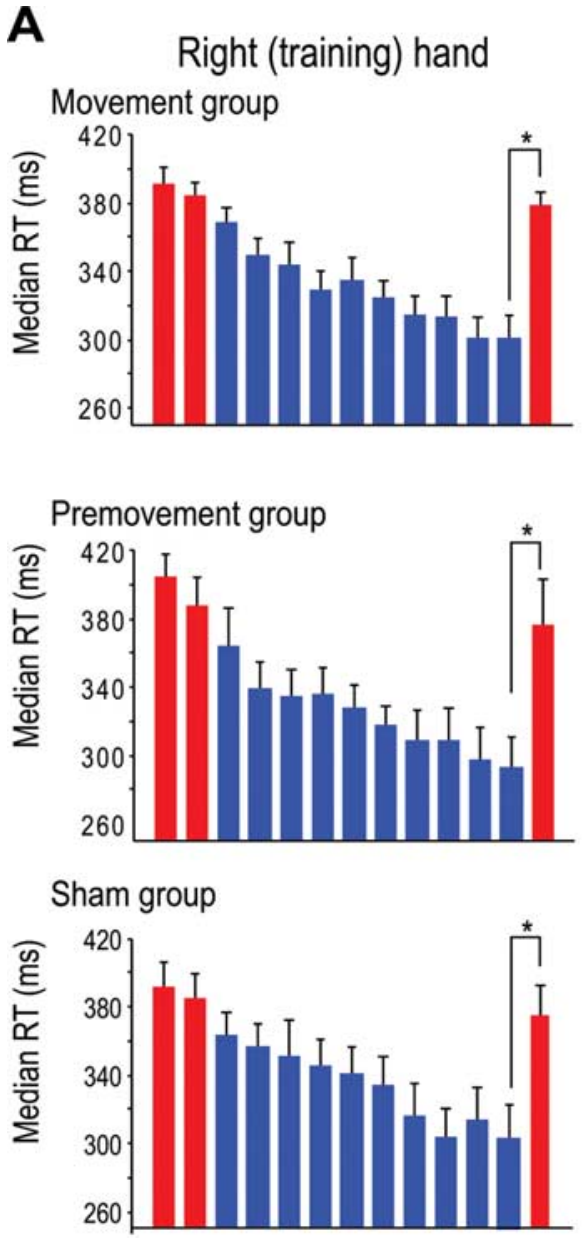

interaction between left-hand block type and testing time $(F=$ $4.1 ; p=0.03)$. Post hoc testing showed that intermanual transfer was larger for the training sequence (blue; $60 \pm 12.3 \mathrm{~ms}$ ) than for the random block (red; $22.8 \pm 9.4 \mathrm{~ms} ; p<0.01$ ). No difference was found between the control sequence and the random block $\Delta \mathrm{RT}(p=0.4)$. Although the control sequence showed lesser improvement (green; $43 \pm 11.1 \mathrm{~ms}$ ) than the training sequence, this difference was not statistically significant $(p=0.09)$. The following data show why this was the case. For the training sequence, RT was stable over the 10 repetitions within the transfertesting block (repetitions 1 and 2, $61 \mathrm{~ms}$; repetitions 3 and 4, 68 ms; repetitions 5 and 6, $56 \mathrm{~ms}$; repetitions 7 and 8, $52 \mathrm{~ms}$; repetitions 9 and $10,64 \mathrm{~ms} ; F=0.24 ; p=0.8$ ). For the control sequence, RT was stable during the first six repetitions of the sequence (repetitions 1 and 2, $26 \mathrm{~ms}$; repetitions 3 and 4, $31 \mathrm{~ms}$; repetitions 5 and $6,36 \mathrm{~ms} ; F=0.36 ; p=0.7$ ) and improved during the later repetitions of the sequence, as would be expected when the subjects practiced a new sequence (repetitions 7 and 8 , $57 \mathrm{~ms}$; repetitions 9 and $10,63 \mathrm{~ms} ; p=0.01$ ). The number of 

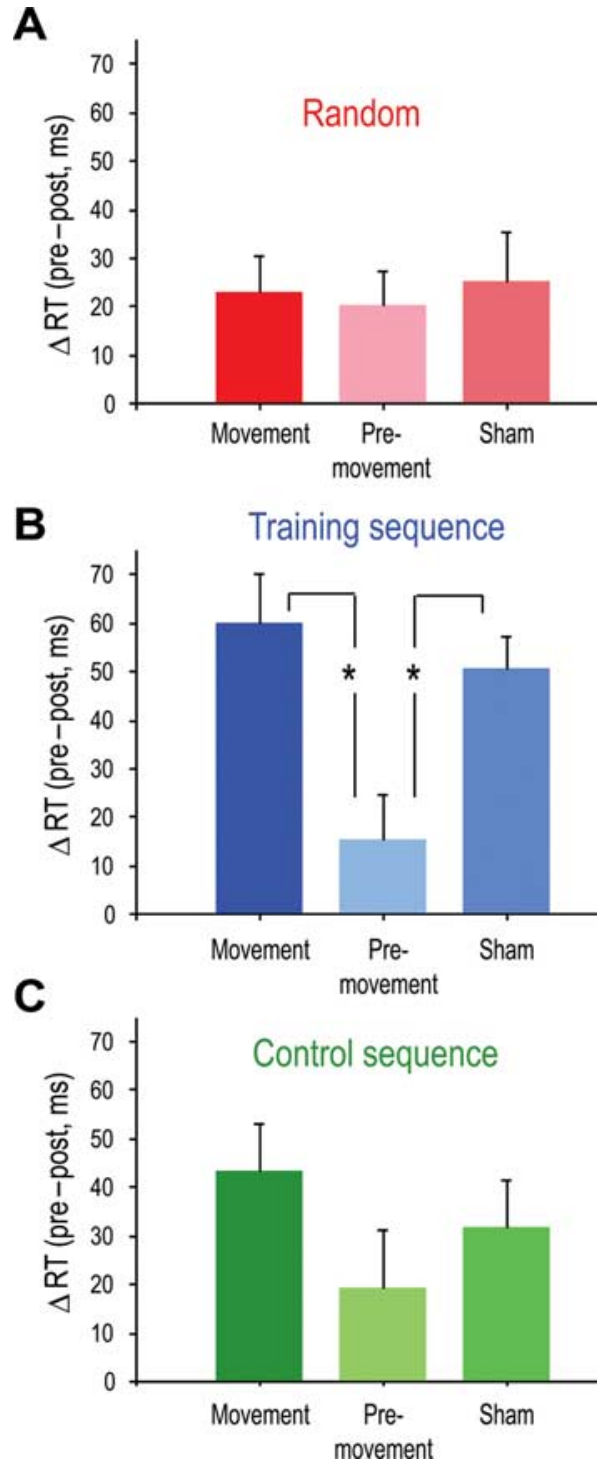

Figure 3. Effect of rTMS over the SMA on intermanual transfer of sequence learning. The abscissa shows the three groups: movement (darkest bar), premovement (brightest bar), and sham; the ordinate shows the difference in RT for the left hand before and after SRTT training (of the other hand) for three types of blocks: random $(\boldsymbol{A})$, trained sequence $(\boldsymbol{B})$, and control sequence $(\boldsymbol{C})$. Note the significantly smaller intermanual transfer for the trained sequence in the premovement group. Variance, SEM.

errors performed with the left hand were comparable across blocks before and after training $\left(\mathrm{ANOVA}_{\mathrm{RM}}, F=0.13, p=0.87\right)$.

For the premovement group, ANOVA $_{\mathrm{RM}}$ showed no significant interaction between left-hand block and testing time $(F=$ $0.26 ; p=0.7)$. In the mirror image version of transfer, no differences were observed in the $\Delta \mathrm{RT}$ in the left hand among the training sequence (15.3 $\pm 10.6 \mathrm{~ms})$, random block $(20.3 \pm 3 \mathrm{~ms})$, and control sequence $(19.0 \pm 12.9 ; F=0.12 ; p=0.8)$. In the goalbased version of transfer, which involves the original training sequence without mirror image inversion, we likewise observed no differences in the $\Delta \mathrm{RT}$ in the left hand among the training sequence $(22.3 \pm 13 \mathrm{~ms})$, random block $(20.8 \pm 6 \mathrm{~ms})$, and control sequence $(16.0 \pm 6.7 ; F=0.12 ; p=0.8)$. The number of errors were comparable across blocks before and after training in both groups, respectively $\left(\mathrm{ANOVA}_{\mathrm{RM}}, F=0.7, p=0.4\right.$; ANO$\mathrm{VA}_{\mathrm{RM}}, F=0.3, p=0.6$ ).

For the sham group involving transfer of the mirror image sequence, ANOVA $_{\mathrm{RM}}$ showed a significant interaction between left-hand block and testing time $(F=4.5 ; p=0.02)$. Post hoc testing showed that the $\Delta \mathrm{RT}$ in the left hand was larger for the training sequence $(50.7 \pm 6.1 \mathrm{~ms})$ than for the random block $(24.9 \pm 12.1 \mathrm{~ms} ; p=0.01)$. The control sequence showed lesser improvements $(31.6 \pm 13.2)$ than the trained sequence, but this difference was not significant $(p=0.07)$. No differences were found between the control sequence and the random block RT $(p=0.6)$ or in the number of errors across blocks (ANOVA $A_{\mathrm{RM}}$, $F=0.02, p=0.8)$. In the sham group involving transfer of the original (non-mirror image) sequence, ANOVA $_{\mathrm{RM}}$ also showed a significant interaction between left-hand block and testing time $(F=6.0 ; p \leq 0.01)$. Post hoc testing showed that the $\Delta \mathrm{RT}$ in the left hand was larger for the training sequence $(57.4 \pm 14 \mathrm{~ms})$ than for the random block $(15.5 \pm 7 \mathrm{~ms} ; p=0.01)$ and control sequence $(26.7 \pm 7.5 \mathrm{~ms} ; p=0.04)$. No differences were found in the number of errors across blocks $\left(\mathrm{ANOVA}_{\mathrm{RM}}, F=0.4, p=0.6\right)$.

When data were grouped according to the left-hand block types (Fig. 3), two-way ANOVA $\mathrm{RM}_{\text {M }}$ showed a significant interaction between stimulation time and left-hand block types $(F=$ $3.11 ; p=0.02$ ). Post hoc one-way ANOVA showed a significant effect of stimulation time on $\Delta \mathrm{RT}$ in the training sequence $(F=$ $5.77 ; p=0.01)$, but not in the control sequence $(F=1.2 ; p=0.3)$ or in random blocks $(F=0.51 ; p=0.6)$. For the training sequence block (Fig. $3 \mathrm{~B}$ ), post hoc testing showed a significant difference between $\Delta \mathrm{RT}$ for rTMS in the premovement versus movement groups $(p \leq 0.01)$ and for the premovement versus sham groups $(p=0.03)$. No RT differences were found between the control sequence and the random block $(p=0.76)$.

\section{Discussion}

Here, we investigated the timing of the contribution of the SMA to successful intermanual transfer of procedural knowledge. We found that a transient virtual lesion over the SMA applied between individual key presses (premovement group) during learning with the right hand substantially interfered with intermanual transfer to the left hand, without disrupting learning. Neither SMA disruption during key press movements (movement group) nor sham stimulation under our experimental design had any effect on intermanual transfer or learning.

Several studies have demonstrated that a motor skill learned with one hand can transfer to the opposite hand (Parlow and Kinsbourne, 1989; Howard and Howard, 1997; Grafton et al., 2002; Japikse et al., 2003; Wang and Sainburg, 2003; Obayashi, 2004; Perez et al., 2007a,b). Cortical areas such as the primary motor cortex, which has relatively sparse interhemispheric connections for the distal musculature (Rouiller et al., 1994), contribute to nonspecific performance improvements in the transfer hand, particularly optimizing the timing of visuomotor processing (Grafton et al., 2002; Perez et al., 2007a), whereas other motor regions, such as the SMA, with strong interhemispheric projections (Liu et al., 2002), play a critical role in successful intermanual transfer of motor sequence skills, both in healthy individuals (Perez et al., 2007b) and in patients with lesions of the corpus callosum (de Guise et al., 1999). Here, we investigated when the SMA makes the contribution to intermanual transfer of procedural sequence knowledge in healthy subjects.

We found that disruption of SMA activity before but not during each individual finger key press or during sham substantially diminished intermanual transfer of knowledge. Previous work showed that activity in SMA neurons encodes the order of components of a motor sequence (Tanji and Shima, 1994). Consistently, in humans, disruption of the SMA before execution affects 
the ability to choose the proper upcoming movements in a motor sequence (Gerloff et al., 1997). Although these studies provided crucial information on the role of the SMA in the preparation of individual movements in a sequence, the timing of SMA contribution to intermanual transfer of knowledge from a learning to a resting hand has not been investigated. Our findings show that this contribution occurs between key presses, during the time when memory of the previous movement (and current hand posture) could contribute to encoding a movement pair. As a sequence is learned, this interval is increasingly involved in preparing and selecting the next movement in the learned sequence. In agreement, studies in monkeys have shown that activity in SMA neurons encodes the order of components of a motor sequence, especially during the period between movements (Tanji and Shima, 1994; Shima and Tanji, 1998, 2000). One possible account for our result, therefore, is that rTMS applied during this preparatory phase interfered with sensorimotor processes, reflected in the preparatory neural activity, that encode the sequence in a form suitable for transfer of knowledge to the opposite hemisphere (Krakauer et al., 2006; Seidler and Noll, 2008).

There are different mechanisms by which the SMA may contribute to intermanual transfer of learning. It is possible that a memory representation stored in the SMA during learning can be accessed by either hand at the time of transfer as proposed by the "access model" of intermanual transfer by Taylor and Heilman (1980). Also, a separate memory can be stored during learning in the contralateral hemisphere, which can be accessed on-line by the transfer hand as proposed by the "cross-activation model" by Parlow and Kinsbourne (1989). Our results of a selective interference of transfer by SMA stimulation support the view that the SMA is involved in encoding a memory trace that can be accessed by both hands, and that this concept can be distinguished from an on-line transfer process that guides the opposite hand. It is important to note that the behavioral effects of rTMS over SMA on intermanual transfer could represent the consequence of focal disruption of activity in the SMA (Gerloff et al., 1997), or its interconnected areas (Lee et al., 2003), and/or disruption of other areas of the brain intending to compensate for the transient SMA deficit.

However, rTMS applied to the SMA during the movement did not disrupt intermanual transfer, suggesting that SMA movement-related activity, which encodes information on movement kinematics and dynamics (Seitz and Roland, 1992; Padoa-Schioppa et al., 2002) and contributes to monitor motor performance (Lee and Quessy, 2003), may play a less critical role in intermanual transfer.

Intermanual transfer of a sequence of events in the SRTT (Nissen and Bullemer, 1987) requires first that participants learn to respond as rapidly as possible to the location of the stimuli. The subjects in our study learned at a slower pace than in previous investigations (Perez et al., 2007a,b), consistent with previous reports we found that the SRTT task performed at slower presentation rates elicits less prominent sequence learning (Willingham et al., 1997; Soetens et al., 2004) than when the task is performed at faster presentation rates (Willingham et al., 1997; Soetens et al., 2004). Importantly, learning of the SRTT under our experimental conditions was comparable across groups, consistent with previous results (Pascual-Leone et al., 1996), although we note that higher rTMS stimulus intensities can disrupt motor learning (Gerloff et al., 1997). In our study, subjects also showed improvements in the control sequence when performing with the left (transfer) hand. Although this improvement was not significantly different from that for the random block, it appears to have resulted mainly from rapid learning of the control sequence during transfer testing. This fast learning may be related to the previous experience with the control sequence that established a baseline level of performance (Fig. 1, left part), some high frequency pairs that are common to the training and control sequence (such as 4-2 and 1-3) (see Materials and Methods), or a possible effect of rTMS during movement on later performance (Avanzino et al., 2008).

Our finding of differential effects of SMA disruption on transfer as opposed to learning is consistent with the view that intermanual transfer and motor learning, although related processes, engage partially different mechanisms (Seidler, 2007; Seidler and Noll, 2008). These findings may also be explained by the model for sequence learning proposed by Hikosaka et al. (1999). These authors suggested that sequence knowledge is acquired by parallel networks, one involved in a goal-based sequence representation (an extrinsic coordinate frame) and another involved in a movement-based sequence representation (an intrinsic coordinate frame). Because the SMA appears to be a critical node in the network guiding the movement-based representation, one possibility is that compensation by the goal-based system may account for the failure of SMA stimulation to affect sequence learning (as opposed to transfer). Furthermore, our observation that rTMS disrupted the intermanual transfer of both the mirror sequence (a movement-based representation) and the original sequence (a goal-based representation), to the same extent, supports the view that the role of the SMA in transfer involves sequence representations that draw on both intrinsic and extrinsic coordinate frames. If, as suggested by the model of Hikosaka et al. (1999), SMA primarily contributes to movement-based representations, then its disruption during learning appears to affect the formation of both goal- and movement-based representations that are available to the controllers of both hands.

\section{Conclusion}

In summary, our results provide novel evidence of a differential and time-specific involvement of the SMA in the successful intermanual transfer of a motor skill. The involvement is differential in that rTMS applied to the SMA during the learning of a motor sequence with one hand does not affect learning, but it does block intermanual transfer of the motor sequence skill indicating that its role in intermanual transfer is at least partially independent of its role in motor learning. The effect is time specific in that the contribution of the SMA is critical during the interval between key presses, when specific movement sequences are encoded.

\section{References}

Avanzino L, Bove M, Trompetto C, Tacchino A, Ogliastro C, Abbruzzese G (2008) 1-Hz repetitive TMS over ipsilateral motor cortex influences the performance of sequential finger movements of different complexity. Eur J Neurosci 27:1285-1291.

de Guise E, del Pesce M, Foschi N, Quattrini A, Papo I, Lassonde M (1999) Callosal and cortical contribution to procedural learning. Brain 122:1049-1062.

Di Lazzaro V, Oliviero A, Pilato F, Saturno E, Dileone M, Mazzone P, Insola A, Tonali PA, Rothwell JC (2004) The physiological basis of transcranial motor cortex stimulation in conscious humans. Clin Neurophysiol 115:255-266.

Elsinger CL, Harrington DL, Rao SM (2006) From preparation to online control: reappraisal of neural circuitry mediating internally generated and externally guided actions. Neuroimage 31:1177-1187.

Gerloff C, Corwell B, Chen R, Hallett M, Cohen LG (1997) Stimulation over the human supplementary motor area interferes with the organization of future elements in complex motor sequences. Brain 120:1587-1602. 
Grafton ST, Hazeltine E, Ivry RB (2002) Motor sequence learning with the nondominant left hand. A PET functional imaging study. Exp Brain Res 146:369-378.

Halsband U, Lange RK (2006) Motor learning in man: a review of functional and clinical studies. J Physiol Paris 99:414-424.

Hikosaka O, Nakahara H, Rand MK, Sakai K, Lu X, Nakamura K, Miyachi S, Doya K (1999) Parallel neural networks for learning sequential procedures. Trends Neurosci 22:464-471.

Howard JH Jr, Howard DV (1997) Age differences in implicit learning of higher order dependencies in serial patterns. Psychol Aging 12:634-656.

Japikse KC, Negash S, Howard JH Jr, Howard DV (2003) Intermanual transfer of procedural learning after extended practice of probabilistic sequences. Exp Brain Res 148:38-49.

Jenkins IH, Brooks DJ, Nixon PD, Frackowiak RS, Passingham RE (1994) Motor sequence learning: a study with positron emission tomography. J Neurosci 14:3775-3790.

Krakauer JW, Mazzoni P, Ghazizadeh A, Ravindran R, Shadmehr R (2006) Generalization of motor learning depends on the history of prior action. PLoS Biol 4:e316.

Lee D, Quessy S (2003) Activity in the supplementary motor area related to learning and performance during a sequential visuomotor task. J Neurophysiol 89:1039-1056.

Lee L, Siebner HR, Rowe JB, Rizzo V, Rothwell JC, Frackowiak RS, Friston KJ (2003) Acute remapping within the motor system induced by lowfrequency repetitive transcranial magnetic stimulation. J Neurosci 23:5308-5318.

Liu J, Morel A, Wannier T, Rouiller EM (2002) Origins of callosal projections to the supplementary motor area (SMA): a direct comparison between pre-SMA and SMA-proper in macaque monkeys. J Comp Neurol 443:71-85.

Macar F, Anton JL, Bonnet M, Vidal F (2004) Timing functions of the supplementary motor area: an event-related fMRI study. Brain Res Cogn Brain Res 21:206-215.

Matsunaga K, Maruyama A, Fujiwara T, Nakanishi R, Tsuji S, Rothwell JC (2005) Increased corticospinal excitability after $5 \mathrm{~Hz}$ rTMS over the human supplementary motor area. J Physiol 562:295-306.

Nakamura K, Sakai K, Hikosaka O (1998) Neuronal activity in medial frontal cortex during learning of sequential procedures. J Neurophysiol 80:2671-2687.

Nissen MJ, Bullemer P (1987) Attentional requirements of learning: evidence from performance measures. Cognit Psychol 19:1-32.

Obayashi S (2004) Possible mechanism for transfer of motor skill learning: implication of the cerebellum. Cerebellum 3:204-211.

Padoa-Schioppa C, Li CS, Bizzi E (2002) Neuronal correlates of kinematicsto-dynamics transformation in the supplementary motor area. Neuron 36:751-765.

Parlow SE, Kinsbourne M (1989) Asymmetrical transfer of training between hands: implications for interhemispheric communication in normal brain. Brain Cogn 11:98-113.

Pascual-Leone A, Wassermann EM, Grafman J, Hallett M (1996) The role of the dorsolateral prefrontal cortex in implicit procedural learning. Exp Brain Res 107:479-485.
Perez MA, Wise SP, Willingham DT, Cohen LG (2007a) Neurophysiological mechanisms involved in transfer of procedural knowledge. J Neurosci 27:1045-1053.

Perez MA, Tanaka S, Wise SP, Sadato N, Tanabe HC, Willingham DT, Cohen LG (2007b) Neural substrates of intermanual transfer of a newly acquired motor skill. Curr Biol 17:1896-1902.

Rand MK, Hikosaka O, Miyachi S, Lu X, Miyashita K (1998) Characteristics of a long-term procedural skill in the monkey. Exp Brain Res 118:293-297.

Rossini PM, Barker AT, Berardelli A, Caramia MD, Caruso G, Cracco RQ, Dimitrijević MR, Hallett M, Katayama Y, Lücking CH (1994) Noninvasive electrical and magnetic stimulation of the brain, spinal cord and roots: basic principles and procedures for routine clinical application. Report of an IFCN committee. Electroencephalogr Clin Neurophysiol 91:79-92.

Rouiller EM, Babalian A, Kazennikov O, Moret V, Yu XH, Wiesendanger M (1994) Transcallosal connections of the distal forelimb representations of the primary and supplementary motor cortical areas in macaque monkeys. Exp Brain Res 102:227-243.

Seidler RD (2007) Aging dissociates motor learning and generalization of learning. Learn Mem 14:17-21.

Seidler RD, Noll DC (2008) Neuroanatomical correlates of motor acquisition and motor transfer. J Neurophysiol 99:1836-1845.

Seitz RJ, Roland PE (1992) Learning of sequential finger movements in man: a combined kinematic and positron emission tomography (PET) study. Eur J Neurosci 4:154-165.

Shibasaki H, Sadato N, Lyshkow H, Yonekura Y, Honda M, Nagamine T, Suwazono S, Magata Y, Ikeda A, Miyazaki M, Fukuyama H, Asato R, Konishi J (1993) Both primary motor cortex and supplementary motor area play an important role in complex finger movement. Brain 116:1387-1398.

Shima K, Tanji J (1998) Both supplementary and presupplementary motor areas are crucial for the temporal organization of multiple movements, J Neurophysiol 80:3247-3260.

Shima K, Tanji J (2000) Neuronal activity in the supplementary and presupplementary motor areas for temporal organization of multiple movements. J Neurophysiol 84:2148-2160.

Soetens E, Melis A, Notebaert W (2004) Sequence learning and sequential effects. Psychol Res 69:124-137.

Tanji J, Shima K (1994) Role for supplementary motor area cells in planning several movements ahead. Nature 371:413-416.

Taylor HG, Heilman KM (1980) Left-hemisphere motor dominance in righthanders. Cortex 16:587-603.

Wang J, Sainburg RL (2003) Mechanisms underlying interlimb transfer of visuomotor rotations. Exp Brain Res 149:520-526.

Willingham DB, Greenberg AR, Thomas RC (1997) Response-to-stimulus interval does not affect implicit motor sequence learning. Mem Cognit 25:534-542.

Willingham DB, Wells LA, Farrell JM, Stemwedel ME (2000) Implicit motor sequence learning is represented in response locations. Mem Cognit 28:366-375. 